\title{
Brazilian Journal \\ of Chemical

\section{EVALUATION OF THE ACTIVATED CHARCOALS AND ADSORPTION CONDITIONS USED IN THE TREATMENT OF SUGARCANE BAGASSE HYDROLYSATE FOR XYLITOL PRODUCTION}

\author{
J. M. Marton ${ }^{1 *}$, M. G. A. Felipe ${ }^{1}$, J. B. Almeida e Silva ${ }^{1}$ and A. Pessoa Júnior ${ }^{2}$ \\ ${ }^{1}$ School of Chemical Engineering at Lorena (FAENQUIL), Department of Biotechnology, \\ Phone: +(55) (12) 3159-5027, Fax: +(55) (12) 3153-3165, \\ P.O. Box: 116, Zip Code 12600-000, Lorena - SP, Brazil \\ E-mail: jmarcelo@cetesb.sp.gov.br \\ ${ }^{2}$ University of São Paulo (USP), São Paulo - SP, Brazil.
}

(Received: May 31, 2004 ; Accepted: August 20, 2005 )

\begin{abstract}
Xylitol has sweetening, anticariogenic and clinical properties that have attracted the attention of the food and pharmaceutical industries. The conversion of sugars from lignocellulosic biomass into xylitol by D-xylose-fermenting yeast represents an alternative to the chemical process for producing this polyol. A good source of D-xylose is sugarcane bagasse, which can be hydrolyzed with dilute acid. However, acetic acid, which is toxic to the yeast, also appears in the hydrolysate, inhibiting microbe metabolism. Xylitol production depends on the initial D-xylose concentration, which can be increased by concentrating the hydrolysate by vacuum evaporation. However, with this procedure the amount of acetic acid is also increased, aggravating the problem of cell inhibition. Hydrolysate treatment with powdered activated charcoal is used to remove or decrease the concentration of this inhibitor, improving xylitol productivity as a consequence. Our work was an attempt to improve the fermentation of Candida guilliermondii yeast in sugarcane bagasse hydrolysate by treating the medium with seven types of commercial powdered activated charcoals (Synth, Carbon Delta A, Carbon Delta G, Carbon 117, Carbon 118L, Carbon 147 and Carvorite), each with its own unique physicochemical properties. Various adsorption conditions were established for the variables temperature, contact time, shaking, $\mathrm{pH}$ and charcoal concentration. The experiments were based on multivariate statistical concepts, with the application of fractional factorial design techniques to identify the variables that are important in the process. Subsequently, the levels of these variables were quantified by overlaying the level curves, which permitted the establishment of the best adsorption conditions for attaining high levels of xylitol volumetric productivity and D-xylose-to-xylitol conversion. This procedure consisted in increasing the original $\mathrm{pH}$ of the hydrolysate to 7.0 with $\mathrm{CaO}$ and reducing it to 5.5 with $\mathrm{H}_{3} \mathrm{PO}_{4}$. Next, the hydrolysate was treated under adsorption conditions employing CDA powdered activated charcoal (1\%) for 30 min at $60^{\circ} \mathrm{C}$, $100 \mathrm{rpm}$ and $\mathrm{pH}$ 2.5. The optimized xylitol volumetric productivity $(0.50 \mathrm{~g} / \mathrm{L} \mathrm{h})$ corresponded to a D-xyloseto-xylitol conversion of $0.66 \mathrm{~g} / \mathrm{g}$.

Keywords: Xylitol; sugarcane bagasse; Powdered activated charcoal; Hemicellulosic hydrolysate; Factorial design; Overlaying plot.
\end{abstract}

\section{INTRODUCTION}

Xylitol, a alcohol very sweet, is an active anticariogenic agent suitable for diabetics and patients needing parental nutrition (Ylikarhri, 1979).
Owing to its physicochemical and technological properties, xylitol can be used as an ingredient in different food products, alone or in combination with other sugars (Hyvönen et al.,1982). Its property of prevention of diseases such as otitis and osteoporosis

*To whom correspondence should be addressed 
is one of the most recent findings concerning the clinical applications of xylitol (Mattila et al., 1998; Uhari et al., 2001). Xylitol can be produced by chemical reduction of D-xylose, but this method involves expensive purification steps that can be avoided by using fermentation (Melaja \& Hamalainen, 1997). More profitable processes are based on acid hydrolysis of the hemicellulosic fraction of some lignocellulosic agro-industrial byproducts, which are selectively converted into Dxylose solutions for use as culture media in bioconversion (Felipe et al., 1997; Sene et al., 2000; Alves et al., 2002).

One of the most abundant lignocellulosic agroindustrial by-products in Brazil is bagasse, a fibrous residue resulting from the crushing of sugarcane stalks to extract their juice. In recent years, there has been an increasing trend towards more efficient utilization of agro-industrial residues, including sugarcane bagasse, which consists of approximately $50 \%$ cellulose, $25 \%$ hemicellulose and 25\% lignin. The chemical constituents of bagasse are $\alpha$-cellulose (about 50\%), pentosans (30\%) and ash (2.4\%). Because of its low ash content, bagasse is more suitable for the microbial bioconversion process than such crop residues as rice straw and wheat straw, whose ash contents are $17.5 \%$ and $11.0 \%$, respectively (Pandey et al., 2000). Hydrolysis of pentosan, a component of hemicellulose that contains more D-xylose than L-arabinose, provides a good substrate for microbial cultivation.

Hydrolytic reactions in dilute-acid medium are very complex, mainly because the substrate is in a solid phase and the catalyst is in a liquid phase. The mechanism of hydrolytic reaction includes: (a) diffusion of protons through the wet lignocellulosic matrix; (b) protonation of the oxygen of a heterocyclic ether bond between the sugar monomers; (c) rupture of the ether bond; (d) generation of a carbocation as an intermediate; (e) solvation of the carbocation with water; (f) regeneration of the proton with cogeneration of the sugar monomer, oligomer or polymer, depending on the position of the ether bond; (g) diffusion of the reaction products in the liquid phase when form and size permit (some large oligomers cannot cross the matrix); and (h) return to the second step to begin again (Herrera et al., 2002).

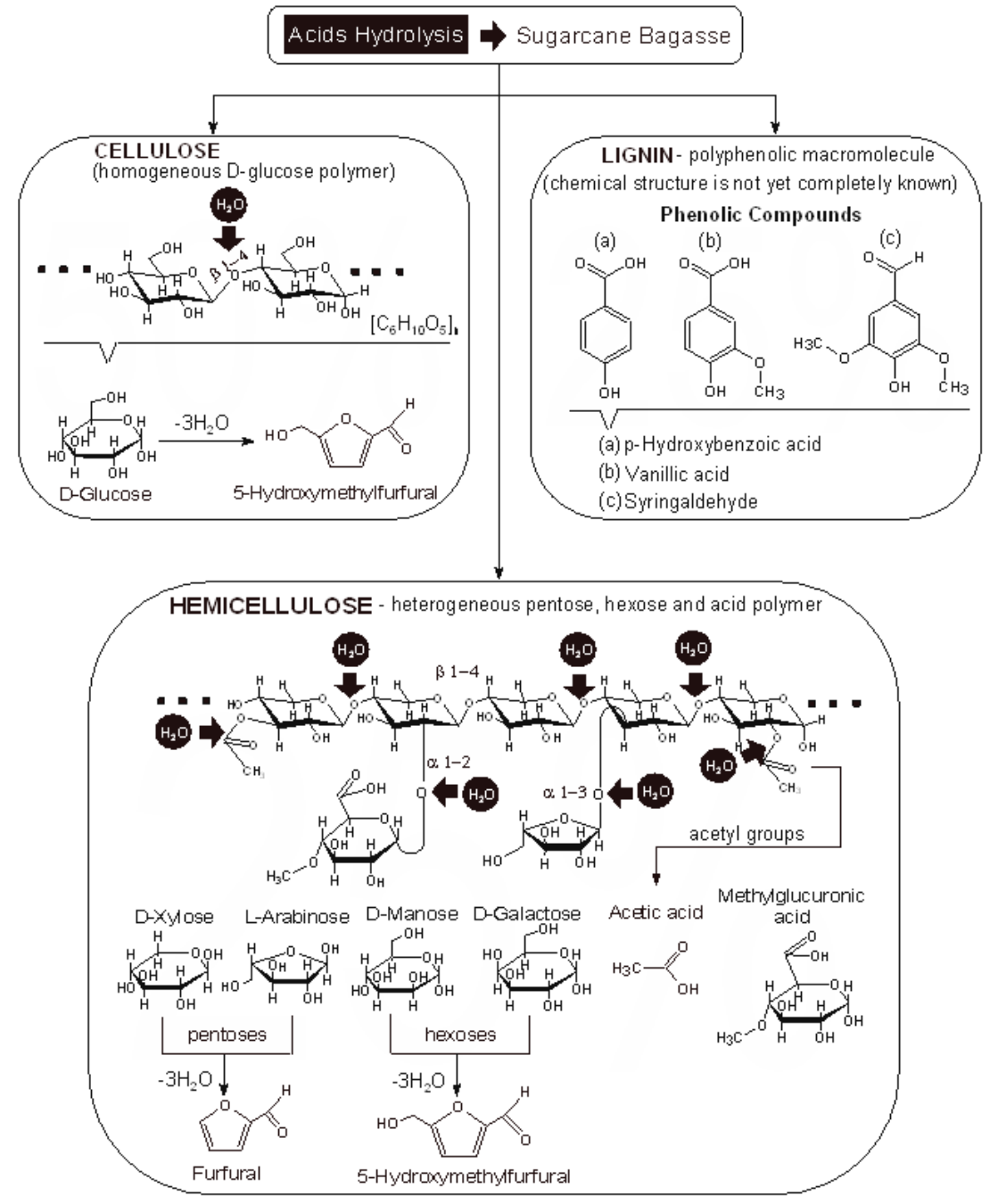

Figure 1: Sketch of sugarcane bagasse degradation by acid hydrolysis 
The main problems in the bioprocess utilizing hydrolysates are the individual and interactive toxic effects of some compounds occurring during the hydrolytic process (Figure 1). These compounds are divided into four groups: (1) substances that are released by the hemicellulosic structure, such as acetic acid, which originates in the deacetylation of xylan; (2) phenolic compounds and other aromatic compounds derived from the partial degradation of lignin; (3) the furan derivatives, furfural and 5-hydroxymethylfurfural, resulting from the degradation of pentoses and hexoses, respectively; and (4) metals like chromium, copper, iron and nickel leached from the equipment (Parajó et al., 1998; Palmqvist et al., 2000). All these toxic compounds need to be eliminated or their concentrations reduced so that the hydrolysates can be effectively used in bioconversion processes.

This work focuses on the treatment of sugarcane bagasse hydrolysate by adjustment of $\mathrm{pH}$ and adsorption on activated charcoal, employing different charcoals under different adsorption conditions in order to improve the bioconversion of D-xylose into xylitol and to obtain empirical models able to describe the interrelationship between the operational adsorption conditions and the fermentative parameters derived from the experimental data.

\section{MATERIAL AND METHODS}

\section{Microorganism and Preparation of Inoculum}

The yeast Candida guilliermondii FTI20037 was used for bioconversion of D-xylose into xylitol. A stock culture, which was maintained at $4^{\circ} \mathrm{C}$ on an agar malt extract slant, was transferred to a $125 \mathrm{~mL}$ Erlenmeyer flask containing $50 \mathrm{~mL}$ liquid medium (30.0g/L D-xylose, 2.0g/L (NH4) ${ }_{2} \mathrm{SO}_{4}, 0.1 \mathrm{~g} / \mathrm{L} \mathrm{CaCl}_{2} .2 \mathrm{H}_{2} \mathrm{O}$ and $20.0 \mathrm{~g} / \mathrm{L}$ rice bran extract solution and incubated at a stirring rate of $200 \mathrm{rpm}$ at $30^{\circ} \mathrm{C}$ for $24 \mathrm{~h}$. The cells were then centrifuged at 2000xg for $15 \mathrm{~min}$ and washed in sterile distilled water. A suspension was prepared with the cell mass in sterile water and utilized as inoculum. For the experiments, the initial cell concentration was $1.0 \mathrm{~g} / \mathrm{L}$ (about $3 \times 10^{7}$ cells $/ \mathrm{mL}$ ).

\section{Preparation and Treatment of Hemicellulose Hydrolysate}

Sugarcane bagasse was introduced into a 350L reactor and mixed with concentrated $\mathrm{H}_{2} \mathrm{SO}_{4}$ (100mg of acid per gram of dry matter) at a solid:liquid ratio of 1:10 (Pessoa Junior et al., 1997) After hydrolysis $\left(121^{\circ} \mathrm{C}, 10 \mathrm{~min}\right)$, the hydrolysate containing $(\mathrm{g} / \mathrm{L}) \mathrm{D}$ glucose (0.84), D-xylose (17.85), L-arabinose (1.68), acetic acid (3.15), phenolic compounds (2.20), furfural (0.06) and 5-hydroxymethylfurfural (0.008) was filtered and concentrated under vacuum at $70^{\circ} \mathrm{C}$ to quadruple the D-xylose concentration. The hydrolysate thus obtained had the following composition (g/L): D-glucose (3.68), D-xylose (69.23), L-arabinose (7.15), acetic acid (6.02), phenolic compounds (7.88), furfural (0.021) and 5hydroxymethylfurfural (0.024). The hydrolysate was treated as follows: the initial $\mathrm{pH}(0.92)$ was raised to 7.0 with commercial $\mathrm{CaO}$ and the hydrolysate was then acidified with concentrated $\mathrm{H}_{3} \mathrm{PO}_{4}$ to $\mathrm{pH} 5.5$ with the subsequent addition of powdered activated charcoal. The charcoal was mixed with the hydrolysate under different adsorption conditions. In all the treatments the precipitates resulting from adjustment of $\mathrm{pH}$ and from addition of activated charcoal were removed by vacuum filtration. For this study seven different types of powdered activated charcoals were used, Synth (Synth, São Paulo, Brazil), CDA and CDG (Brasilac, Paraná, Brazil), C117, C118L and C147 (Carbomafra, Paraná, Brazil) and Carvorite (Carvorite, Paraná, Brazil). These treatments resulted in several hydrolysates that were autoclaved at $111^{\circ} \mathrm{C}$ for $15 \mathrm{~min}$, before they were used as culture media.

\section{Medium and Fermentation Conditions}

The hydrolysates obtained from the treatment were supplemented with nutrients the same as those, used in inoculum preparation with the exception of D-xylose. Fermentations were carried out in $125 \mathrm{~mL}$ Erlenmeyer flasks containing $50 \mathrm{~mL}$ of culture medium ( $\mathrm{pH}$ 5.5) on a rotatory shaker at 200rpm and $30^{\circ} \mathrm{C}$ for $64 \mathrm{~h}$.

\section{Analytical Methods}

The concentrations of D-glucose, L-arabionse, Dxylose, xylitol, acetic acid, furfural and 5- 
hydroxymethylfurfural were determined by highperformance liquid chromatrography (Rodrigues et al., 2001), and the phenolic concentration by the colorimetric method using $\mathrm{FeCl}_{3} \cdot 6 \mathrm{H}_{2} \mathrm{O}$ and $\mathrm{K}_{3} \mathrm{Fe}(\mathrm{CN})_{6}$ at $700 \mathrm{~nm}$ (Kim \& Yoo, 1996). The cell number was determined directly by counting in a Neubauer chamber (area $=1 / 400 \mathrm{~mm}^{2}$; height $=$ $0.100 \mathrm{~mm})$.

\section{Statistical Analysis}

In order to minimize the number of experiments and model the xylitol yield and volumetric productivity, a $2^{5-2}$ fractionary factorial design (Box et al., 1978) was used. This design also allowed us to evaluate the effects of the independent variables temperature, contact time, shaking, $\mathrm{pH}$ and activated charcoal concentration on xylitol yield and volumetric productivity, using the response surface regression procedure. Table 1 shows these variables and their respective maximum $(+1)$ and minimum $(-1)$ coded levels. For statistical calculation, each independent variable was coded according to Eq. (1), where $x_{i}$ is the coded value, $X_{i}$ is the real value, $X_{0}$ is the real value at the center point and $\Delta X_{i}$ is the step change value. The fermentation parameters of the D-xylose-to-xylitol bioconversion, namely xylitol volumetric productivity $\left(\mathrm{Q}_{\mathrm{P}}\right)$ and yield, were taken as the dependent variables or responses of the design experiments.

$\mathrm{x}_{\mathrm{i}}=\frac{\mathrm{X}_{\mathrm{i}}-\mathrm{X}_{0}}{\Delta \mathrm{X}_{\mathrm{i}}}$

The experiments were performed in an aleatory order to minimize systematic errors. After statistical analysis, the variables with significant effects were used to compose a $2^{3}$ full factorial design with a centered face and three replicates at the center point. The statistical analyses of the data were performed using the STATGRAPHICS statistical software, version 4.1 and the STATISTICA program, version 5.0. The results were expressed in estimated effects, standard errors, Student's $t$ distribution tables and tables of analysis of variance (ANOVA) (degree of freedom, sum of squares, mean squares, $F$ - value (Fischer variance ratio) and significance level ( $P$ - value).

\section{RESULTS AND DISCUSSION}

In order to evaluate the efficacy of each powdered activated charcoal employed in the treatment of sugarcane hemicellulosic hydrolysate, a $2^{5-2}$ fractional factorial design in seven blocks was conducted using xylitol volumetric productivity (Qp) as response. The choice of this fermentation parameter for this analysis was due to the fact that low values have been reported for Qp in recent studies (Rodrigues et al., 2003). In Table 1 the experimental matrix is present as are the values of xylitol volumetric productivity provided by $C$. guilliermondii as a function of different adsorption conditions employed in the treatment of sugarcane bagasse hydrolysate. As the one-way ANOVA (Table 2) showed that there was no significant difference $(P<0.05)$ between the blocks, the choice of type of powdered activated charcoal was based on price. The types with the best prices were CDA and C117, so the two blocks represented by CDA and C117 were again submitted to analysis of estimated effects (Table 3 ) in order to select the most appropriate for the experiments. According to Table 3, there was no significant difference between the blocks $(P=0.5952)$ represented by $\mathrm{CDA}$ and $\mathrm{C} 117$, but CDA was chosen because it has a higher iodine number (minimum of $800 \mathrm{mg} / \mathrm{g}$ ), a physical property that measures the adsorption capacity of activated charcoals, than C117 (minimum of $700 \mathrm{mg} / \mathrm{g}$ ). According to Brasilac (n.d.) and Carbomafra (n.d.), iodine number is an important physicochemical property that shows by correlation the adsorption surface area of the activated charcoal; it indicates that the larger the iodine number the larger the adsorption area. Danner et al. (2003) verified that physicochemical properties of activated charcoal affect the removal of inhibitors such as furan and phenolic compounds from wood hydrolysates for improving the fermentability of this hydrolysate, observing that hydrolysates treated with activated charcoals prepared at a higher temperature $\left(1000^{\circ} \mathrm{C}\right)$ could attain higher fermentability than charcoal prepared at a lower temperature $\left(400^{\circ} \mathrm{C}\right)$. 
Table 1: Experimental matrix of a $2^{5-2}$ fractional factorial design in blocks for xylitol volumetric productivity as a function of operational conditions for adsorption with coded and natural levels of variables.

\begin{tabular}{|c|c|c|c|c|c|c|c|c|c|c|c|c|c|}
\hline & & & \multicolumn{5}{|c|}{ Variables } & \multicolumn{3}{|c|}{ Coded variables } & \multicolumn{3}{|c|}{ Qp } \\
\hline & Trial & $\operatorname{Run}^{a}$ & $\mathbf{T}^{\mathrm{b}}\left({ }^{\circ} \mathrm{C}\right)$ & $\mathbf{c t}^{\mathrm{c}}(\mathrm{min})$ & $S^{d}(\mathbf{r p m})$ & $\mathbf{p H}$ & $\begin{array}{l}C^{C C^{e}} \\
(\%)\end{array}$ & $\mathbf{x}_{1}{ }^{\mathrm{f}}$ & $\mathbf{x}_{2}{ }^{\mathrm{f}}$ & $\mathbf{x}_{3}{ }^{\mathbf{f}}$ & $\mathbf{x}_{4}{ }^{\mathrm{f}}$ & $\mathbf{X}_{5}{ }^{f}$ & $\begin{array}{c}\text { (g/L.h) } \\
y^{g}\end{array}$ \\
\hline \multirow{8}{*}{ 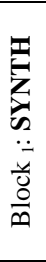 } & 1 & 7 & 30 & 60 & 100 & 1 & 10 & -1 & +1 & -1 & -1 & +1 & 0.37 \\
\hline & 2 & 6 & 60 & 60 & 300 & 6 & 10 & +1 & +1 & +1 & +1 & +1 & 0.43 \\
\hline & 3 & 8 & 60 & 60 & 100 & 6 & 1 & +1 & +1 & -1 & +1 & -1 & 0.38 \\
\hline & 4 & 4 & 30 & 30 & 100 & 6 & 10 & -1 & -1 & -1 & +1 & +1 & 0.42 \\
\hline & 5 & 2 & 60 & 30 & 300 & 1 & 10 & +1 & -1 & +1 & -1 & +1 & 0.43 \\
\hline & 6 & 3 & 30 & 60 & 300 & 1 & 1 & -1 & +1 & +1 & -1 & -1 & 0.35 \\
\hline & 7 & 5 & 30 & 30 & 300 & 6 & 1 & -1 & -1 & +1 & +1 & -1 & 0.47 \\
\hline & 8 & 1 & 60 & 30 & 100 & 1 & 1 & +1 & -1 & -1 & -1 & -1 & 0.39 \\
\hline \multirow{8}{*}{ 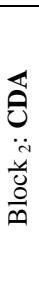 } & 9 & 2 & 60 & 30 & 300 & 1 & 10 & +1 & -1 & +1 & -1 & +1 & 0.50 \\
\hline & 10 & 4 & 30 & 30 & 100 & 6 & 10 & -1 & -1 & -1 & +1 & +1 & 0.44 \\
\hline & 11 & 8 & 60 & 60 & 100 & 6 & 1 & +1 & +1 & -1 & +1 & -1 & 0.35 \\
\hline & 12 & 6 & 60 & 60 & 300 & 6 & 10 & +1 & +1 & +1 & +1 & +1 & 0.40 \\
\hline & 13 & 1 & 60 & 30 & 100 & 1 & 1 & +1 & -1 & -1 & -1 & -1 & 0.54 \\
\hline & 14 & 7 & 30 & 60 & 100 & 1 & 10 & -1 & +1 & -1 & -1 & +1 & 0.33 \\
\hline & 15 & 5 & 30 & 30 & 300 & 6 & 1 & -1 & -1 & +1 & +1 & -1 & 0.43 \\
\hline & 16 & 3 & 30 & 60 & 300 & 1 & 1 & -1 & +1 & +1 & -1 & -1 & 0.29 \\
\hline \multirow{8}{*}{ 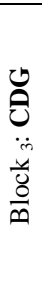 } & 17 & 8 & 60 & 60 & 100 & 6 & 1 & +1 & +1 & -1 & +1 & -1 & 0.37 \\
\hline & 18 & 3 & 30 & 60 & 300 & 1 & 1 & -1 & +1 & +1 & -1 & -1 & 0.31 \\
\hline & 19 & 1 & 60 & 30 & 100 & 1 & 1 & +1 & -1 & -1 & -1 & -1 & 0.48 \\
\hline & 20 & 6 & 60 & 60 & 300 & 6 & 10 & +1 & +1 & +1 & +1 & +1 & 0.43 \\
\hline & 21 & 2 & 60 & 30 & 300 & 1 & 10 & +1 & -1 & +1 & -1 & +1 & 0.49 \\
\hline & 22 & 4 & 30 & 30 & 100 & 6 & 10 & -1 & -1 & -1 & +1 & +1 & 0.41 \\
\hline & 23 & 7 & 30 & 60 & 100 & 1 & 10 & -1 & +1 & -1 & -1 & +1 & 0.45 \\
\hline & 24 & 5 & 30 & 30 & 300 & 6 & 1 & -1 & -1 & +1 & +1 & -1 & 0.45 \\
\hline \multirow{8}{*}{$\begin{array}{l}\text { 금 } \\
\ddot{u} \\
\dot{y} \\
\frac{0}{\text { D }}\end{array}$} & 25 & 8 & 60 & 60 & 100 & 6 & 1 & +1 & +1 & -1 & +1 & -1 & 0.37 \\
\hline & 26 & 6 & 60 & 60 & 300 & 6 & 10 & +1 & +1 & +1 & +1 & +1 & 0.43 \\
\hline & 27 & 3 & 30 & 60 & 300 & 1 & 1 & -1 & +1 & +1 & -1 & -1 & 0.32 \\
\hline & 28 & 7 & 30 & 60 & 100 & 1 & 10 & -1 & +1 & -1 & -1 & +1 & 0.38 \\
\hline & 29 & 4 & 30 & 30 & 100 & 6 & 10 & -1 & -1 & -1 & +1 & +1 & 0.42 \\
\hline & 30 & 5 & 30 & 30 & 300 & 6 & 1 & -1 & -1 & +1 & +1 & -1 & 0.44 \\
\hline & 31 & 1 & 60 & 30 & 100 & 1 & 1 & +1 & -1 & -1 & -1 & -1 & 0.51 \\
\hline & 32 & 2 & 60 & 30 & 300 & 1 & 10 & +1 & -1 & +1 & -1 & +1 & 0.48 \\
\hline \multirow{8}{*}{ 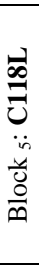 } & 33 & 7 & 30 & 60 & 100 & 1 & 10 & -1 & +1 & -1 & -1 & +1 & 0.38 \\
\hline & 34 & 8 & 60 & 60 & 100 & 6 & 1 & +1 & +1 & -1 & +1 & -1 & 0.37 \\
\hline & 35 & 2 & 60 & 30 & 300 & 1 & 10 & +1 & -1 & +1 & -1 & +1 & 0.49 \\
\hline & 36 & 1 & 60 & 30 & 100 & 1 & 1 & +1 & -1 & -1 & -1 & -1 & 0.54 \\
\hline & 37 & 5 & 30 & 30 & 300 & 6 & 1 & -1 & -1 & +1 & +1 & -1 & 0.52 \\
\hline & 38 & 4 & 30 & 30 & 100 & 6 & 10 & -1 & -1 & -1 & +1 & +1 & 0.44 \\
\hline & 39 & 3 & 30 & 60 & 300 & 1 & 1 & -1 & +1 & +1 & -1 & -1 & 0.35 \\
\hline & 40 & 6 & 60 & 60 & 300 & 6 & 10 & +1 & +1 & +1 & +1 & +1 & 0.39 \\
\hline \multirow{8}{*}{ 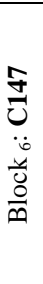 } & 41 & 4 & 30 & 30 & 100 & 6 & 10 & -1 & -1 & -1 & +1 & +1 & 0.45 \\
\hline & 42 & 2 & 60 & 30 & 300 & 1 & 10 & +1 & -1 & +1 & -1 & +1 & 0.53 \\
\hline & 43 & 6 & 60 & 60 & 300 & 6 & 10 & +1 & +1 & +1 & +1 & +1 & 0.44 \\
\hline & 44 & 8 & 60 & 60 & 100 & 6 & 1 & +1 & +1 & -1 & +1 & -1 & 0.35 \\
\hline & 45 & 1 & 60 & 30 & 100 & 1 & 1 & +1 & -1 & -1 & -1 & -1 & 0.56 \\
\hline & 46 & 7 & 30 & 60 & 100 & 1 & 10 & -1 & +1 & -1 & -1 & +1 & 0.41 \\
\hline & 47 & 5 & 30 & 30 & 300 & 6 & 1 & -1 & -1 & +1 & +1 & -1 & 0.49 \\
\hline & 48 & 3 & 30 & 60 & 300 & 1 & 1 & -1 & +1 & +1 & -1 & -1 & 0.28 \\
\hline \multirow{8}{*}{ 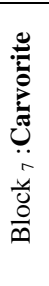 } & 49 & 2 & 60 & 30 & 300 & 1 & 10 & +1 & -1 & +1 & -1 & +1 & 0.55 \\
\hline & 50 & 4 & 30 & 30 & 100 & 6 & 10 & -1 & -1 & -1 & +1 & +1 & 0.42 \\
\hline & 51 & 6 & 60 & 60 & 300 & 6 & 10 & +1 & +1 & +1 & +1 & +1 & 0.46 \\
\hline & 52 & 3 & 30 & 60 & 300 & 1 & 1 & -1 & +1 & +1 & -1 & -1 & 0.32 \\
\hline & 53 & 7 & 30 & 60 & 100 & 1 & 10 & -1 & +1 & -1 & -1 & +1 & 0.45 \\
\hline & 54 & 1 & 60 & 30 & 100 & 1 & 1 & +1 & -1 & -1 & -1 & -1 & 0.50 \\
\hline & 55 & 5 & 30 & 30 & 300 & 6 & 1 & -1 & -1 & +1 & +1 & -1 & 0.52 \\
\hline & 56 & 8 & 60 & 60 & 100 & 6 & 1 & +1 & +1 & -1 & +1 & -1 & 0.36 \\
\hline
\end{tabular}

${ }^{\mathrm{a}}$ Run: sequence of experiments

${ }^{\mathrm{b}} \mathrm{T}$ : temperature; ${ }^{\mathrm{C}}$ tc: contact time ; ${ }^{\mathrm{d}} \mathrm{S}$ : shaking; ${ }^{\mathrm{e}} \mathrm{CC}$ : activated charcoal concentration (w/v)

${ }^{\mathrm{f}} \mathrm{x}_{1}, \mathrm{x}_{2}, \mathrm{x}_{3}, \mathrm{x}_{4}$ and $\mathrm{x}_{5}$ are dimensionless, normalized and independent variables

$\mathrm{g}$ : dependent variable (volumetric productivity) 
Table 2: One-way ANOVA for xylitol volumetric productivity obtained with C. guilliermondii cultivated in sugarcane bagasse hydrolysate using a $2^{5-2}$ fractional factorial design in two blocks (CDA and C117).

\begin{tabular}{|l|c|c|c|c|c|}
\hline Source of variation & Sum of squares & Df $^{\text {a }}$ & Mean squares & F-ratio & P-value \\
\hline Between blocks & 0.01145 & 6 & 0.00191 & 0.376 & 0.8905 \\
Within blocks & 0.24842 & 49 & 0.00507 & & \\
Total (corrected) & 0.25987 & 55 & & & \\
\hline
\end{tabular}

${ }^{\mathrm{a}}$ Degrees of freedom

Table 3: Estimates, standard errors and Student's $t$ test for xylitol volumetric productivity obtained with $C$. guilliermondii cultivated in sugarcane bagasse hydrolysate using a $2^{5-2}$ fractional factorial design in two blocks (CDA and C117).

\begin{tabular}{|c|c|c|c|}
\hline Effects & Estimates & Standard errors & \multicolumn{1}{c|}{-value } \\
\hline Average & 0.6338 & \pm 0.0126 & - \\
$\mathrm{X}_{1}$ & 0.0500 & \pm 0.0252 & $-7.9841^{\mathrm{a}}$ \\
$\mathrm{X}_{2}$ & -0.1875 & \pm 0.0252 & $-7405^{\mathrm{a}}$ \\
$\mathrm{X}_{3}$ & 0.0525 & \pm 0.0252 & $2.0834^{\mathrm{a}}$ \\
$\mathrm{X}_{4}$ & -0.0650 & \pm 0.0252 & $-2.5794^{\mathrm{a}}$ \\
$\mathrm{X}_{5}$ & -0.0150 & \pm 0.0252 & -0.5952 \\
$\mathrm{X}_{2} \mathrm{X}_{3}+\mathrm{x}_{4} \mathrm{x}_{5}$ & \pm 0.0252 & 1.0913 \\
$\mathrm{X}_{2} \mathrm{x}_{5}+\mathrm{x}_{3} \mathrm{X}_{4}$ & -0.0275 & \pm 0.0252 & 0.1984 \\
Between blocks & 0.0050 & \pm 0.0252 & 0.5952 \\
\hline
\end{tabular}

${ }^{\mathrm{a}}$ confidence level of $90 \%\left(t_{10 \%}=1.753\right)$

Analysis of the estimated effect for xylitol volumetric productivity (Table 3) shows that temperature, contact time, shaking and $\mathrm{pH}$ had significant effects $(P<0.10)$. As the effect of charcoal concentration (CC) was not significant $(P>0.10)$, this variable was eliminated and other experiments were conducted with a view to attaining a $2^{4}$ full factorial design using the responses xylitol volumetric productivity (Qp) and yield ( $\mathrm{Y}_{\mathrm{P} / \mathrm{S}}$ ) (Table 4).

Table 4 contains the experimental matrix as well as the results achieved for the fermentation parameters of the D-xylose-to-xylitol bioconversion by $C$. guilliermondii as a function of different adsorption conditions employed in the treatment of sugarcane bagasse hemicellulosic hydrolysate. After the $2^{4}$ full factorial design, the fermentation parameters were analyzed by response surface methodology. Analysis of the estimated effects (Table 5) shows that temperature, contact time and interaction between temperature and $\mathrm{pH}$ had significant effects $(P<0.05)$ on xylitol volumetric productivity (Qp). With respect to yield, temperature, contact time $\mathrm{pH}$, interaction between temperature and contact time and interaction between temperature and $\mathrm{pH}$ also had significant effects $(P<0.05)$. Neither the shaking parameter nor its interactions with other variables had significant effects on yield and xylitol volumetric productivity; thus treatment at the lower level (100 rpm) was preferred, because it consumes less energy.

A $2^{3}$ full factorial design with three replicates at center point was used to obtain the mathematical model representing this fermentation process by the response surface methodology. The response values used in runs 1 to 8 were the average of the runs at the same level for the parameters temperature, contact time and $\mathrm{pH}$ of the $2^{4}$ full factorial design. The matrix for this design and for the fermentation parameters ( $\mathrm{Qp}$ and $\left.\mathrm{Y}_{\mathrm{P} / \mathrm{S}}\right)$ is shown in Table 6. Firstly an ANOVA of the experimental data was conducted to estimate the quadratic model. This analysis showed that for xylitol volumetric productivity (Qp), the curvature was significant at the $10 \%$ probability level $(P<0.10)$.

However, this same analysis for D-xylose-toxylitol yield did not have a significant effect at the $10 \%$ probability level $(P>0.10)$ (data not shown). For these reasons, the xylitol volumetric productivity experiments were combined (Table 7), obtaining a $2^{3}$ full factorial design with a centered face and three replicates at the center point.

As is evident in Table 7 , run 2 provided the highest xylitol volumetric productivity (0.52 g/L.h), whereas run 3 provided the lowest productivity (0.31 g/L.h). This difference is equivalent to $40 \%$. As for D-xylose-to-xylitol yield Table 6 shows that the variation between maximum and minimum values $(0.77 \mathrm{~g} / \mathrm{g}$ for run 2 and $0.51 \mathrm{~g} / \mathrm{g}$ for run 8) corresponded to a decrease of $34 \%$, which confirms that the different adsorption conditions employed in the treatment of sugarcane bagasse hydrolysate all had an effect on the D-xylose-to-xylitol bioconversion. 
Table 4: Experimental matrix for xylitol volumetric productivity $\left(Q_{P}\right)$ and yield $\left(Y_{P / S}\right)$ obtained with $C$. guilliermondii cultivated in sugarcane bagasse hydrolysate treated with CDA activated charcoal at natural and coded levels, using a $2^{4}$ full factorial design with three replicates at the center point.

\begin{tabular}{|c|c|c|c|c|c|c|c|c|c|c|c|}
\hline \multirow[b]{3}{*}{ Trial $^{\mathrm{a}}$} & \multirow[b]{3}{*}{$\operatorname{Run}^{\mathbf{b}}$} & \multirow[b]{2}{*}{$\mathbf{T}\left({ }^{\circ} \mathbf{C}\right)^{\mathrm{C}}$} & \multirow[b]{2}{*}{$\operatorname{tc}(\min )^{d}$} & \multicolumn{2}{|c|}{ Variables } & \multicolumn{4}{|c|}{ Coded variables } & \multicolumn{2}{|c|}{ Responses } \\
\hline & & & & $\mathrm{S}(\mathbf{r p m})^{\mathrm{e}}$ & $\mathbf{p H}$ & $\mathbf{x}_{1}{ }^{\mathbf{f}}$ & $\mathbf{x}_{2}{ }^{\mathbf{f}}$ & $\mathbf{x}_{3}{ }^{\mathrm{f}}$ & $\mathbf{x}_{4}{ }^{\mathbf{f}}$ & Qp & $\mathbf{Y}_{\mathbf{P} / \mathrm{S}}$ \\
\hline & & $\mathbf{x}_{1}$ & $\mathbf{x}_{2}$ & $\mathbf{x}_{3}$ & $\mathbf{x}_{4}$ & & & & & (g/L.h) & (g/g) \\
\hline \multirow{3}{*}{10} & 1 & 60 & 30 & 300 & 6.0 & +1 & -1 & +1 & +1 & 0.39 & 0.59 \\
\hline & & 30 & 30 & 100 & 6.0 & -1 & -1 & -1 & +1 & 0.44 & 0.62 \\
\hline & 2 & 30 & 30 & 100 & 1.0 & -1 & -1 & -1 & -1 & 0.37 & 0.56 \\
\hline 15 & & 30 & 30 & 300 & 6.0 & -1 & -1 & +1 & +1 & 0.43 & 0.66 \\
\hline \multirow[t]{2}{*}{11} & & 60 & 60 & 100 & 6.0 & +1 & +1 & -1 & +1 & 0.35 & 0.52 \\
\hline & 3 & 60 & 60 & 300 & 1.0 & +1 & +1 & +1 & -1 & 0.43 & 0.59 \\
\hline \multirow[t]{3}{*}{16} & & 30 & 60 & 300 & 1.0 & -1 & +1 & +1 & -1 & 0.29 & 0.58 \\
\hline & 4 & 30 & 30 & 300 & 1.0 & -1 & -1 & +1 & -1 & 0.38 & 0.58 \\
\hline & 5 & 30 & 60 & 300 & 6.0 & -1 & +1 & +1 & +1 & 0.43 & 0.61 \\
\hline \multirow[t]{2}{*}{12} & & 60 & 60 & 300 & 6.0 & +1 & +1 & +1 & +1 & 0.40 & 0.50 \\
\hline & 6 & 60 & 60 & 100 & 1.0 & +1 & +1 & -1 & -1 & 0.39 & 0.58 \\
\hline 09 & & 60 & 30 & 300 & 1.0 & +1 & -1 & +1 & -1 & 0.50 & 0.78 \\
\hline \multirow[t]{2}{*}{13} & & 60 & 30 & 100 & 1.0 & +1 & -1 & -1 & -1 & 0.54 & 0.77 \\
\hline & 7 & 60 & 30 & 100 & 6.0 & +1 & -1 & -1 & +1 & 0.44 & 0.58 \\
\hline \multirow[t]{5}{*}{14} & & 30 & 60 & 100 & 1.0 & -1 & +1 & -1 & -1 & 0.33 & 0.58 \\
\hline & 8 & 30 & 60 & 100 & 6.0 & -1 & +1 & -1 & +1 & 0.39 & 0.57 \\
\hline & 9 & 45 & 45 & 200 & 3.5 & 0 & 0 & 0 & 0 & 0.43 & 0.59 \\
\hline & 10 & 45 & 45 & 200 & 3.5 & 0 & 0 & 0 & 0 & 0.42 & 0.61 \\
\hline & 11 & 45 & 45 & 200 & 3.5 & 0 & 0 & 0 & 0 & 0.44 & 0.59 \\
\hline
\end{tabular}

${ }^{\mathrm{a}}$ Experiments from $2^{5-2}$ fractional factorial design

${ }^{\mathrm{b}}$ Run: sequence of experiments

${ }^{\mathrm{c}} \mathrm{T}$ : temperature, ${ }^{\mathrm{d}}$ tc: contact time and ${ }^{\mathrm{e}} \mathrm{S}$ : shaking

${ }^{\mathrm{f}} \mathrm{x}_{1}, \mathrm{x}_{2}, \mathrm{x}_{3}, \mathrm{x}_{4}$ and $\mathrm{x}_{5}$ are dimensionless, normalized and independent variables

${ }^{\mathrm{g}} \mathrm{y}_{1}$ and $\mathrm{y}_{2}$ : dependent variables (xylitol volumetric productivity and yield, respectively)

Table 5: Effect estimates, standard errors and t-test for xylitol productivity and yield in the D-xylose-to-xylitol bioconversion obtained with C. guilliermondii, using a $2^{4}$ full factorial design with three replicates at the center point.

\begin{tabular}{|c|c|c|c|c|c|c|}
\hline \multirow[b]{2}{*}{ Effects } & \multirow[b]{2}{*}{ Estimates } & \multicolumn{2}{|c|}{ Productivity (Qp) } & \multirow[b]{2}{*}{ Estimates } & \multirow{2}{*}{$\begin{array}{c}\text { Yield }\left(\mathbf{Y}_{\mathrm{P} / \mathrm{S}}\right) \\
\text { Standard errors }\end{array}$} & \multirow[b]{2}{*}{$t$-values } \\
\hline & & Standard errors & $t$-values & & & \\
\hline Average & 0.410 & \pm 0.006 & - & 0.603 & \pm 0.007 & - \\
\hline $\mathrm{x}_{1}$ & 0.047 & \pm 0.013 & $3.615^{\mathrm{a}}$ & 0.187 & \pm 0.017 & $11.000^{\mathrm{a}}$ \\
\hline $\mathrm{x}_{2}$ & -0.060 & \pm 0.013 & $-4.615^{a}$ & -0.076 & \pm 0.017 & $-4.471^{\mathrm{a}}$ \\
\hline $\mathrm{x}_{3}$ & 0.000 & \pm 0.013 & 0.000 & 0.014 & \pm 0.017 & 0.823 \\
\hline$x_{4}$ & 0.005 & \pm 0.013 & 0.385 & -0.046 & \pm 0.017 & $-2.706^{\mathrm{a}}$ \\
\hline $\mathrm{x}_{1} \mathrm{x}_{2}$ & -0.015 & \pm 0.013 & 1.154 & -0.056 & \pm 0.017 & $-3.294^{\mathrm{a}}$ \\
\hline $\mathrm{x}_{1} \mathrm{x}_{3}$ & 0.000 & \pm 0.013 & 0.000 & -0.011 & \pm 0.017 & 0.647 \\
\hline $\mathrm{x}_{1} \mathrm{x}_{4}$ & -0.075 & \pm 0.013 & $5.769^{\mathrm{a}}$ & -0.086 & \pm 0.017 & $-5.059^{\mathrm{a}}$ \\
\hline $\mathrm{x}_{2} \mathrm{X}_{3}$ & 0.023 & \pm 0.013 & 1.769 & -0.006 & \pm 0.017 & -0.353 \\
\hline $\mathrm{x}_{2} \mathrm{x}_{4}$ & 0.027 & \pm 0.013 & 2.077 & 0.014 & \pm 0.017 & 0.823 \\
\hline $\mathrm{x}_{3} \mathrm{x}_{4}$ & 0.007 & \pm 0.013 & 0.538 & 0.004 & \pm 0.017 & 0.235 \\
\hline
\end{tabular}

${ }^{\mathrm{a}}$ Significant at a probability level of $5 \%\left(t_{5 \%}=2.131\right)$ 
Table 6: Experimental matrix for xylitol volumetric productivity $\left(Q_{P}\right)$ and yield $\left(Y_{P / S}\right)$ obtained with $C$. guilliermondii cultivated in sugarcane bagasse hydrolysate treated with CDA activated charcoal at natural and coded levels, using a $2^{3}$ full factorial design with three replicates at the center point.

\begin{tabular}{|c|c|c|c|c|c|c|c|c|}
\hline & \multicolumn{3}{|c|}{ Variables } & \multicolumn{3}{|c|}{ Coded variables } & \multicolumn{2}{|c|}{ Responses } \\
\hline & $\begin{array}{c}\text { Temperature } \\
\left({ }^{\circ} \mathrm{C}\right)\end{array}$ & $\begin{array}{c}\text { Contact } \\
\text { time (min) }\end{array}$ & $\mathbf{p H}$ & $\mathbf{x}_{1}$ & $\mathbf{x}_{2}$ & $\mathbf{x}_{4}$ & $\mathbf{Q}_{\mathbf{P}}$ & $\mathbf{Y}_{\mathbf{P} / \mathbf{S}}$ \\
\hline Run & $\mathrm{x}_{1}$ & $\mathrm{x}_{2}$ & $\mathbf{x}_{4}$ & & & & $\mathrm{y}_{1}{ }^{\mathrm{a}}$ & $y_{2}{ }^{a}$ \\
\hline 1 & 30 & 30 & 1.0 & -1 & -1 & -1 & 0.37 & 0.57 \\
\hline 2 & 60 & 30 & 1.0 & +1 & -1 & -1 & 0.52 & 0.77 \\
\hline 3 & 30 & 60 & 1.0 & -1 & +1 & -1 & 0.31 & 0.58 \\
\hline 4 & 60 & 60 & 1.0 & +1 & +1 & -1 & 0.41 & 0.59 \\
\hline 5 & 30 & 30 & 6.0 & -1 & -1 & +1 & 0.43 & 0.64 \\
\hline 6 & 60 & 30 & 6.0 & +1 & -1 & +1 & 0.41 & 0.59 \\
\hline 7 & 30 & 60 & 6.0 & -1 & +1 & +1 & 0.41 & 0.59 \\
\hline 8 & 60 & 60 & 6.0 & +1 & +1 & +1 & 0.37 & 0.51 \\
\hline 9 & 45 & 45 & 3.5 & 0 & 0 & 0 & 0.43 & 0.59 \\
\hline 10 & 45 & 45 & 3.5 & 0 & 0 & 0 & 0.42 & 0.61 \\
\hline 11 & 45 & 45 & 3.5 & 0 & 0 & 0 & 0.44 & 0.59 \\
\hline
\end{tabular}

${ }^{\mathrm{a}} \mathrm{y}_{1}$ and $\mathrm{y}_{2}$ : dependent variables (xylitol volumetric productivity and yield, respectively)

Table 7: Experimental matrix for xylitol volumetric productivity $\left(Q_{P}\right)$ obtained with C. guilliermondii cultivated in sugarcane bagasse hydrolysate treated with CDA activated charcoal at natural and coded levels, using a $2^{3}$ full factorial design with centered face and three replicates at the center point.

\begin{tabular}{|c|c|c|c|c|c|c|c|}
\hline \multirow[b]{2}{*}{ Run } & \multicolumn{3}{|c|}{ Variables } & \multicolumn{3}{|c|}{ Coded variables } & \multirow{2}{*}{$\begin{array}{c}\text { Response } \\
\begin{array}{c}Q_{P}(g / L . h \\
y_{1}\end{array}\end{array}$} \\
\hline & $\begin{array}{c}\text { Temperature } \\
\left({ }^{\circ} \mathrm{C}\right) \\
\mathrm{x}_{1} \\
\end{array}$ & $\begin{array}{c}\text { Contact time } \\
\text { (min) } \\
x_{2} \\
\end{array}$ & $\begin{array}{c}\text { pH } \\
\mathbf{x}_{4}\end{array}$ & $\mathbf{x}_{1}$ & $\mathbf{x}_{2}$ & $\mathbf{x}_{4}$ & \\
\hline 1 & 30 & 30 & 1.0 & -1 & -1 & -1 & 0.37 \\
\hline 2 & 60 & 30 & 1.0 & +1 & -1 & -1 & 0.52 \\
\hline 3 & 30 & 60 & 1.0 & -1 & +1 & -1 & 0.31 \\
\hline 4 & 60 & 60 & 1.0 & +1 & +1 & -1 & 0.41 \\
\hline 5 & 30 & 30 & 6.0 & -1 & -1 & +1 & 0.43 \\
\hline 6 & 60 & 30 & 6.0 & +1 & -1 & +1 & 0.41 \\
\hline 7 & 30 & 60 & 6.0 & -1 & +1 & +1 & 0.41 \\
\hline 8 & 60 & 60 & 6.0 & +1 & +1 & +1 & 0.37 \\
\hline $9^{a}$ & 30 & 45 & 3.5 & -1 & 0 & 0 & 0.42 \\
\hline $10^{\mathrm{a}}$ & 60 & 45 & 3.5 & +1 & 0 & 0 & 0.49 \\
\hline $11^{\mathrm{a}}$ & 45 & 30 & 3.5 & 0 & -1 & 0 & 0.46 \\
\hline $12^{\mathrm{a}}$ & 45 & 60 & 3.5 & 0 & +1 & 0 & 0.48 \\
\hline $14^{\mathrm{a}}$ & 45 & 45 & 6.0 & 0 & 0 & +1 & 0.36 \\
\hline 15 & 45 & 45 & 3.5 & 0 & 0 & 0 & 0.43 \\
\hline 16 & 45 & 45 & 3.5 & 0 & 0 & 0 & 0.42 \\
\hline 17 & 45 & 45 & 3.5 & 0 & 0 & 0 & 0.44 \\
\hline
\end{tabular}

${ }^{\mathrm{a}}$ runs in the centered face experiments 
The residual analysis obtained by plotting the residuals versus the values estimated by the proposed model showed that the residuals varied randomly, which means they did not have unexplained systematic tendencies, so the curve represented the points satisfactorily (data not shown). Application of the response surface methodology, on the basis of the coefficient estimate, provides an empirical relationship between the values of xylitol volumetric productivity $\left(\mathrm{y}_{1}\right)$ and the coded variables tested $\left(\mathrm{x}_{\mathrm{i}}\right)$ by means of regression equation 2 by replacing the qualitative variable $\mathrm{x}_{2}$ by its lowest coded level (-1):

$\hat{\mathrm{y}}_{1}=0.477+0.026 \mathrm{x}_{1}-0.0218 \mathrm{x}_{4}-$

$-0.0361 \mathrm{x}_{1} \mathrm{x}_{4}-0.0482 \mathrm{x}_{4}^{2}$

where $\mathrm{y}_{1}$ represents the xylitol volumetric productivity (Qp), $\mathrm{x}_{1}$ the initial coded temperature and $\mathrm{x}_{4}$ the coded $\mathrm{pH}$. Solving this mathematical model by means of the coded levels for the temperature $60^{\circ} \mathrm{C}(+1)$ and the $\mathrm{pH} 2.5(-0.4)$ makes it possible to predict $0.518 \mathrm{~g} / \mathrm{L}$ h of xylitol volumetric productivity. The model's optimal region can be observed in Figure 2, which depicts the response surface and contour lines described by the $\mathrm{y}_{1}$ model. When temperature level $+1\left(60^{\circ} \mathrm{C}\right)$ decreases to -1 $\left(30^{\circ} \mathrm{C}\right)$ and the $\mathrm{pH}$ level -1 (1.0) increases to +1 (6.0), xylitol volumetric productivity decreases by 25 and $18 \%$, respectively (Figure 2).

In Table 9 the regression coefficients, standard errors, $t$-values and significance level for the model representing the D-xylose-to-xylitol yield at a significance level of $5 \%$ are presented. In this case, contact time $\left(\mathrm{x}_{2}\right)$, interaction between temperature and contact time and interaction between temperature and $\mathrm{pH}$ had significant effects.

Table 8: Regression coefficients, standard errors, $t$-test and significance levels for the model representing the xylitol volumetric productivity obtained with $C$. guilliermondii, using a $2^{3}$ factorial design with centered face and three replicates at the center point.

\begin{tabular}{|c|c|c|c|c|}
\hline Variables & Coefficients & Standard errors & $\boldsymbol{t}$-values & $\boldsymbol{P}$-values \\
\hline Constant & 0.4477 & \pm 0.0125 & - & - \\
$\mathrm{X}_{1}$ & 0.0260 & \pm 0.0105 & $2.9213^{\mathrm{a}}$ & $0.0130^{\mathrm{a}}$ \\
$\mathrm{X}_{4}$ & -0.0218 & \pm 0.0117 & $-2.4494^{\mathrm{a}}$ & $0.0310^{\mathrm{a}}$ \\
$\mathrm{X}_{1} \mathrm{x}_{4}$ & -0.0361 & \pm 0.0099 & $-3.6331^{\mathrm{a}}$ & $0.0035^{\mathrm{a}}$ \\
$\mathrm{X}_{4} \mathrm{x}_{4}$ & -0.0482 & \pm 0.0164 & $-3.4676^{\mathrm{a}}$ & $0.0047^{\mathrm{a}}$ \\
\hline
\end{tabular}

${ }^{a}$ a confidence level of the $95 \%\left(t_{5 \circ}=2.201\right)$

$\mathrm{R}_{\text {adjusted }}^{2}=0.69$

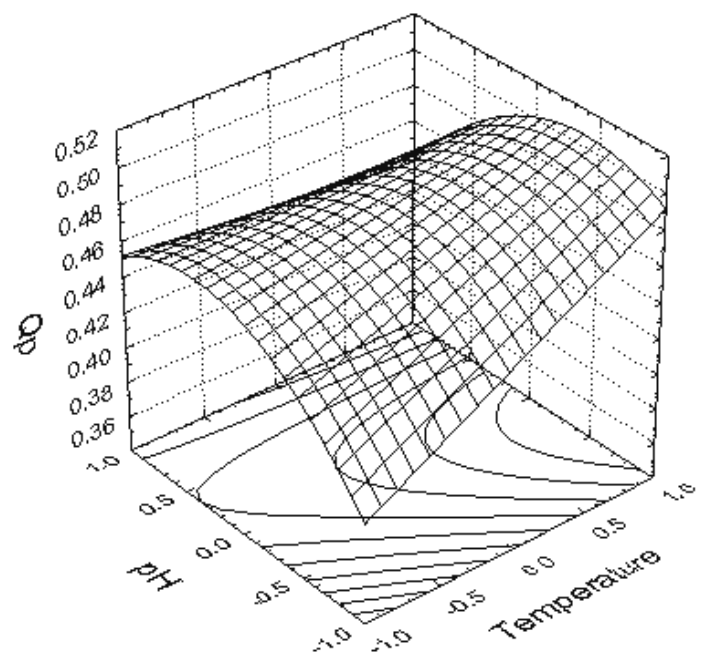

Figure 2: Response surface and contour lines described by the $\mathrm{y}_{1}$ model representing the xylitol volumetric productivity obtained with C. guilliermondii in sugarcane bagasse hemicellulosic hydrolysate. 
Table 9: Regression coefficients, standard errors, $t$-test and significance levels for the model representing the D-xylose-to-xylitol yield obtained with $C$. guilliermondii using a $2^{3}$ factorial design with centered face and three replicates at the center point.

\begin{tabular}{|c|c|c|c|}
\hline Effects & Estimates & Standard errors & t-values \\
\hline Average & 0.6036 & \pm 0.0096 & - \\
$\mathrm{X}_{1}$ & 0.0225 & \pm 0.0226 & 0.9955 \\
$\mathrm{X}_{2}$ & -0.0775 & \pm 0.0226 & $3.4292^{\mathrm{a}}$ \\
$\mathrm{X}_{4}$ & -0.0475 & \pm 0.0226 & 2.1018 \\
$\mathrm{X}_{1} \mathrm{X}_{2}$ & -0.0575 & \pm 0.0226 & $2.5442^{\mathrm{a}}$ \\
$\mathrm{X}_{1} \mathrm{X}_{4}$ & -0.0875 & \pm 0.0226 & $3.8717^{\mathrm{a}}$ \\
$\mathrm{X}_{2} \mathrm{X}_{4}$ & 0.0125 & \pm 0.0226 & 0.5531 \\
\hline
\end{tabular}

${ }^{\mathrm{a}}$ confidence level of $95 \%\left(t_{5 \%}=2.228\right)$

In this case, the residual analysis of the proposed model showed that the curve also represented the points satisfactorily (data not shown). Table 10 shows the ANOVA regression and the model's correlation coefficient $\left(\mathrm{R}^{2}=0.62\right)$ reveals that the mathematical model is significant and can be expressed by equation (3):

$\hat{\mathrm{y}}_{2}=0.60364-0.03875 \mathrm{x}_{2}-$

$-0.0575 \mathrm{x}_{1} \mathrm{x}_{2}-0.04375 \mathrm{x}_{1} \mathrm{x}_{4}$

where $\mathrm{y}_{2}$ represents the D-xylose-to-xylitol yield $\left(\mathrm{Y}_{\mathrm{P} / \mathrm{S}}\right), \mathrm{x}_{2}$ the initial coded contact time and $\mathrm{x}_{1} \mathrm{x}_{4}$ the coded interaction between temperature and $\mathrm{pH}$. Solving this mathematical model by means of the coded levels for contact time 30min $(+1)$, temperature $60^{\circ} \mathrm{C}(+1)$ and $\mathrm{pH} 1.0(-1)$ makes it possible to predict a xylitol yield of $0.69 \mathrm{~g} / \mathrm{g}$. The model's optimal region can be observed in Figure 3 , which depicts the response surface and contour lines described by the $y_{2}$ of the model. According to Figure 3, when the contact time level +1 (60min) decreases to -1 (30min) and the $\mathrm{pH}$ level +1 (6.0) decreases to -1 (1.0), the D-xylose-toxylitol yield increases by 19 and 33\%, respectively.

Table 10: Regression coefficients, standard errors, $t$-test and significance levels for the model representing the xylitol volumetric productivity obtained with $C$. guilliermondii, using a $2^{3}$ factorial design with three replicates at the center point.

\begin{tabular}{|c|c|c|c|c|}
\hline Variables & Coefficients & Standard errors & $\boldsymbol{t}$-values & $\boldsymbol{P}$-values \\
\hline Constant & 0.60364 & \pm 0.013705 & 44.0440 & 0.0000 \\
$\mathrm{X}_{2}$ & -0.03875 & \pm 0.016071 & -2.4112 & $0.0424^{\mathrm{a}}$ \\
$\mathrm{X}_{1} \mathrm{X}_{4}$ & -0.04375 & \pm 0.016071 & -2.7223 & $0.0262^{\mathrm{a}}$ \\
\hline
\end{tabular}

${ }^{\mathrm{a}}$ confidence level of $95 \%\left(t_{5 \%}=2.228\right)$

$\mathrm{R}^{2}=0.62$

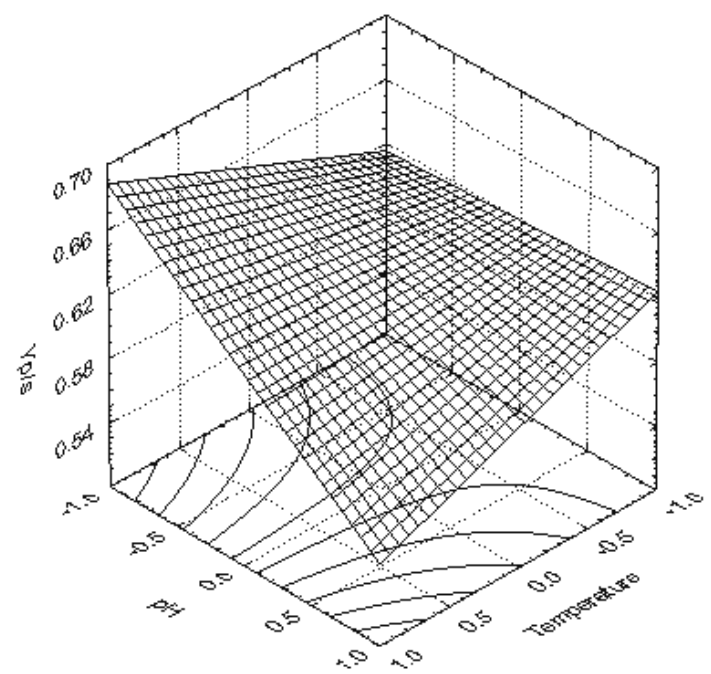

Figure 3: Response surface and contour lines described by the $\mathrm{y}_{2}$ model representing the yield of D-xylose-toxylitol bioconversion obtained with C. guilliermondii in sugarcane bagasse hemicellulosic hydrolysate. 
That both xylitol volumetric productivity (Table 8) and D-xylose-to-xylitol yield obtained (Table 10) shows that the variables temperature and $\mathrm{pH}$ were significant at a confidence level of 95\%. These two variables were used to determine the optimization condition, which resulted in the following models:

$$
\begin{aligned}
& \text { Qp: } \hat{\mathrm{y}}_{1}=0.477+0.026 \mathrm{x}_{1}-0.0218 \mathrm{x}_{4}- \\
& -0.0361 \mathrm{x}_{1} \mathrm{x}_{4}-0.0482 \mathrm{x}_{4}{ }^{2} \\
& \mathrm{Y}_{\mathrm{P} / \mathrm{S}}: \hat{\mathrm{y}}_{2}=0.64239-0.04375 \mathrm{x}_{1} \mathrm{x}_{4}
\end{aligned}
$$

The xylitol volumetric productivity (Qp) is the same as that utilized to plot Figure 2, but in the xylitol yield model the variable $\mathrm{x}_{2}$ (contact time) was substituted for -1 , since this variable has a negative effect at a confidence level of 95\% (Table 9), and the value was added to the constant of the model. Based on the two models (Equations 4 and 5), a graphic optimization was conducted using the Design-Expert program. This method consists in overlaying the curves of all the modes according to the criteria adopted. The optimal working conditions to attain high levels of xylitol volumetric productivity and Dxylose-to-xylitol yield were defined using the following criteria: (1) xylitol volumetric productivity higher than $0.45 \mathrm{~g} / \mathrm{L} \mathrm{h}$ and (2) D-xylose-to-xylitol yield higher than $0.60 \mathrm{~g} / \mathrm{g}$. The overlaying plot in Figure 4 shows a shaded area where the requirements were satisfied. Therefore, a point corresponding to temperature level $+1\left(60^{\circ} \mathrm{C}\right)$ and $\mathrm{pH}$ level $-0.4(2.5)$ was assigned as an optimum point. Under these conditions, the model predicted a xylitol volumetric productivity of $0.51 \mathrm{~g} / \mathrm{L} \mathrm{h}$ (with variation from 0.49 to $0.53 \mathrm{~g} / \mathrm{L} \mathrm{h}$ possible) and a D-xylose-to-xylitol yield of $0.66 \mathrm{~g} / \mathrm{g}$ (with variation from 0.65 to 0.67 $\mathrm{g} / \mathrm{g}$ possible) in the confidence interval of $95 \%$.

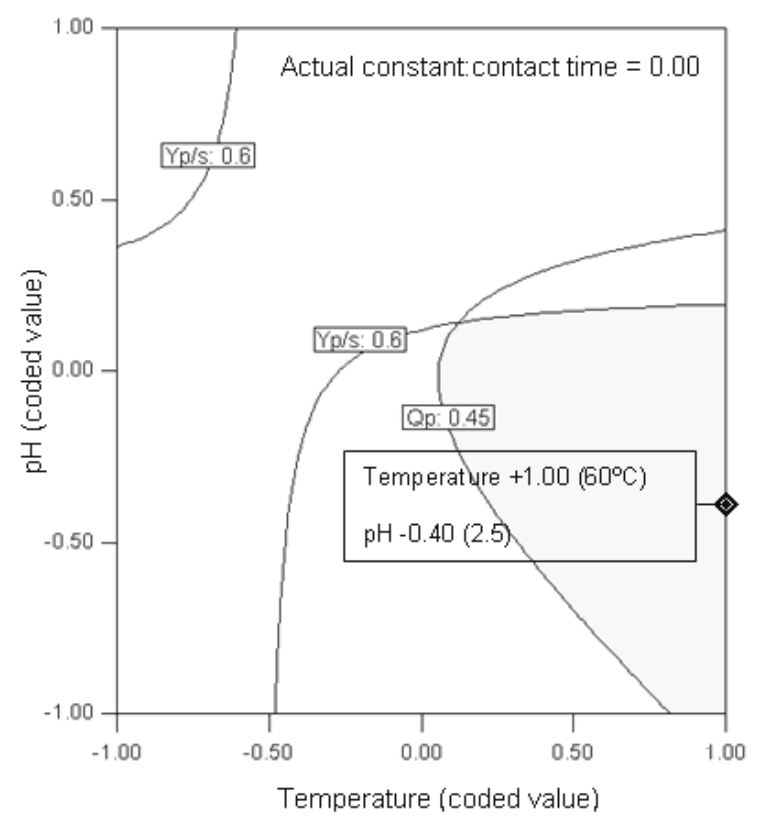

Figure 4: Optimum region obtained by overlaying plots of the two responses ( $\mathrm{Y}_{\mathrm{P} / \mathrm{S}}$ and $\mathrm{Qp}$ ) as a function of temperature and $\mathrm{pH}$.

To verify these optimized conditions the variables temperature, shaking, agitation, $\mathrm{pH}$ and charcoal concentration were fixed at levels +1.00 $\left(60^{\circ} \mathrm{C}\right),-1.00$ (30 min), -1.00 (100 rpm), -0.40 (2.5) and $-1.00(1 \%)$, respectively, and treatments employing these adsorption conditions were conducted in triplicate. The average values of xylitol volumetric productivity and D-xylose-to-xylitol yield were $0.50 \mathrm{~g} / \mathrm{L} \mathrm{h}$ and $0.66 \mathrm{~g} / \mathrm{g}$, respectively. These results showed that the model the experimental data well and thus described well the region under study. Under optimized conditions an average of $76 \%$ of phenolic compounds, $44 \%$ acetic acid, $60 \%$ 5hydroxymethylfurfural and $58 \%$ furfural were removed, and after $64 \mathrm{~h}$ of fermentation $94 \%$ Dxylose, $65 \%$ acetic acid and 55\% L-arabinose had been consumed. D-xylose consumption (94\%) was similar, as also reported by Dominguez et al. (1996) (95\%), who cultivated a mutant of Candida sp 11-2 in sugarcane bagasse hydrolysate treated with activated charcoal to form xylitol. The partial consumption of L-arabinose $(7.15 \mathrm{~g} / \mathrm{L})$ and total consumption of D-glucose, in the hydrolysate at low concentrations (3.68 g/L), were detected in all 
experiments (data not shown), as also reported by Alves et al. (1998) and Rodrigues et al., (2003), who cultivated the same strain in sugarcane bagasse hydrolysate.

In relation to adsorption conditions, the highest temperature $\left(60^{\circ} \mathrm{C}\right)$ employed favored the improvement of fermentation parameters. However, in the study of adsorption operational conditions for improvement of wood hydrolysate fermentability Parajó et al. (1996b) verified that the effect of activated charcoal concentration is about three times higher than that of temperature on D-xylose-toxylitol conversion $\left(\mathrm{Y}_{\mathrm{P} / \mathrm{S}}\right)$. According to these authors, the media treated with an intermediate (205 $\mathrm{g} / \mathrm{g}$ ) or low (400 g/g) hydrolysate:charcoal ratio at $24^{\circ} \mathrm{C}$ were sufficient for $87-88 \%$ D-xylose consumption in $36 \mathrm{~h}$, with volumetric consumption rates in the range of 0.93 to $0.98 \mathrm{~g} / \mathrm{L} \mathrm{h}$ and obtained the maximum xylitol yield $(0.6 \mathrm{~g} / \mathrm{g})$. In this work, the results permitted verification that increasing charcoal concentration, and consequently improving the adsorption surface, favors the desirable removal of all toxic compounds studied, but the highest toxic compound removal was not a proportional to improvement of the fermentation parameters. The highest values for the toxic compound removal, 99\% phenolics (trial 33), 58\% acetic acid (trial 01), 100\% furfural (trial 14) and 100\% 5-hydroxymetylfurfural (trial 01) (data not shown) were obtained with a $10 \%$ concentration of activated charcoal, while xylitol volumetric productivity for these trials was 0.38 , $0.37,0.33$ and $0.37 \mathrm{~g} / \mathrm{L}$ h, respectively (Table 1 ).

With regard to contact time, the fixed value of 30 min was the same as that in a study on wood hydrolysate treatment with activated charcoal; Parajó et al. (1996b) reported that irrespective of the hydrolysate:charcoal ratio, $400 \mathrm{~g} / \mathrm{g}$ or $20 \mathrm{~g} / \mathrm{g}$, the maximum phenol adsorption on activated charcoal occurred in about $30 \mathrm{~min}$ of contact time. The same authors observed that, after a fast initial period of up to about $30 \mathrm{~min}$ and a slow stage, the surface area of the activated charcoal was progressively blocked during the process.

Table 6 shows that for the lowest $\mathrm{pH}$ improved the fermentation parameters (trial 02) related to the reduction of the concentrations of acetic acid and phenols under this condition. According to Rodrigues et al. (2001), the removal of acetic acid $\left(\mathrm{pK}_{\mathrm{a}}=4.76\right)$ by adsorption of activated charcoal is related to the balance between its dissociated and undissociated forms regulated by equilibrium, which is affected by $\mathrm{pH}$. These authors achieved maximum values for removal of acetic acid (62\%) and phenols (98\%) when activated charcoal $(2.4 \% \mathrm{w} / \mathrm{v})$ was added to a fourfold concentration of sugarcane bagasse hydrolysate at $\mathrm{pH}$ 0.92. A possible explanation for the highest values for removal of the toxic acids such as acetic acid and phenolics can be due to their molecular form at a low $\mathrm{pH}$, favoring the London forces (adsorption forces).

\section{CONCLUSIONS}

This study shows the application of statistical theories to fermentation processes and proves that treatment of sugarcane bagasse hydrolysate affects the formation of xylitol. The different types of powdered activated charcoals employed did not have a significant effect on the xylitol volumetric productivity obtained with Candida guilliermondii. The fermentation parameters of the bioconversion of D-xyloste to xylitol were affect by the adsorption conditions employed during treatment of the sugarcane bagasse hydrolysate. The variables temperature, contact time and $\mathrm{pH}$ had significant effects on the parameters xylitol volumetric productivity and xylitol yield. Defining an optimum point made it possible to achieve a xylitol volumetric productivity of $0.50 \mathrm{~g} / \mathrm{L} \mathrm{h}$ and a xylitol yield of 0.66 $\mathrm{g} / \mathrm{g}$. Although the xylitol volumetric productivity improved, a much better result could have been achieved if a more effective method of treatment had been employed. Further work will thus be carried out to evaluate the treatment of sugarcane bagasse hydrolysate in a continuous system employing ionexchange resins and activated charcoal in columns to be used as media in fermentation with adapted cells.

\section{ACKNOWLEDGEMENTS}

The authors gratefully acknowledge the financial support of FAPESP and CNPq. They are also grateful to Maria Eunice M. Coelho for revising this paper.

\section{REFERENCES}

Alves, L.A., Felipe, M.G.A., Almeida e Silva, J.B., Silva, S.S., Prata, A.M.R. Pretreatment of Sugar Cane Bagasse Hemicellulose Hydrolysate for Xylitol Production by Candida guilliermondii. Applied Biochemistry and Biotechnology,1998, 70/2, 89-98.

Alves, L.A., Felipe, M.G.A., Almeida e Silva, J.B. Xylose Reductase and Xylitol Dehydrogenase Activities of Candida guilliermondii as a Function of Different Treatments of Sugarcane 
Bagasse Hemicellulosic Hydrolysate Employing Experimental Design. Applied Biochemistry and Biotechnology, 2002, 98-100, 403-413.

Brasilac - Indústria Química Ltda. Carvão Ativo: um Instrumento do Nosso Cotidiano. GuarapuavaPR, n.d.

Box, G.E., Hunter, W.G., Hunter, J.S. Statistics for Experiments: An Introduction to Design, Data Analysis, and Model Building. New York: Jonh Wiley \& Sons, 1978, 653p.

Carbomafra - Indústria Química S.A. Informativo: Carvão Ativo. Curitiba-PR, n.d.

Danner, H., Miyafuji, H., Neureiter, M., Thomasser, C., Bvochora, J., Szolar, O., Braum, R. Detoxification of Wood Hydrolysates with Wood Charcoal for Increasing the Fermentability of Hydrolysates. Enzyme and Microbial Technology, 2003, 32, 396-400.

Dominguez, J.M., Gong, C.S., Tsao, G.T. Pretreatment of Sugar Cane Bagasse Hemicellulose Hydrolysate for Xylitol Production by Yeast. Applied Biochemistry and Biotechnology, 1996, 57/58, 49-56.

Felipe, M.G.A., Vitolo, M., Mancilha, I.M., Silva, S.S. Fermentation of Sugar Cane Bagasse Hemicellulosic Hydrolysate for Xylitol Production: Effect of pH. Biomass and Bioenergy, 1997, 13,11-14.

Herrera, A., Téllez-Luist, S.J., Ramírez, J.A., Vásques, M. Production of Xylose from Sorghum Straw Using Hydrochloric Acid. Journal of Cereal Science, 2002, 510, 01-08.

Hyvönen, L., Koivistoinen, P., Voirol, F. Food Techonological Evaluation of Xylitol. Advances in Food Research, 1982, 27, 373-403.

Kim, Y., Yoo, Y. Peroxidase Production from Carrot Hairy Root Cell Culture. Enzyme and Microbial Technology, 1996, 18, 553-535.

Mattila, P.T., Knuuttila, M.L.E., Svanberg, M.J. Dietary Xylitol Supplementation Prevents Osteoporosis Changes in Streptozotocin Diabetic Rats. Metabolism, 1998, 4, 578-583.

Melaja, A.J., Hamalainen, L. Process for Making Xylitol - USN 4.008.285, 1997.

Palmqvist, E., Hahn-Hägerdal, B. Fermentation of Lignocellulosic Hydrolysates. I: Inhibition and Detoxification. Bioresource Technology, 2000, 74, 17-24.
Pandey, A., Soccol, C.R., Nigam, P., Socool, V.T. Biotechnological Potential of Agro-industrial Residues. I: Sugarcane Bagasse. Bioresourse Technology, 2000, 74, 69-80.

Parajó, J.C., Domingues, H., Domingues, I.M. Charcoal Adsorption of Wood Hydrolysate for Improving their Fermentability: Influence of the Operational Conditions. Bioresource Technology, 1996a, 57, 179-185.

Parajó, J.C., Domingues, H., Domingues, I.M. Study of Charcoal Adsorption for Improving the Production of Xylitol from Wood Hydrolysates. Bioprocess Engineering, 1996b, 16, 39-43.

Parajó, J.C., Dominguez, H., Dominguez, J.M. Biotechnological Production of Xylitol. Part 3: Operation in Culture Media Made from Lignocellulose Hydrolysate. Bioresource Technology, 1998, 66, 25-40.

Pessoa Junior, A., Mancilha, I.M., Sato, S. Acid Hydrolysis of Hemicellulose from Sugarcane Bagasse. Brazilian Journal of Chemical Engineering, 1997,14, 291-297.

Rodrigues, R.C.L.B., Felipe, M.G.A., Almeida e Silva, J.B., Vitolo, M., Gómez, P.V. The Influence of $\mathrm{pH}$, Temperature and Hydrolysate Concentration on the Removal of Volatile and Nonvolatile Compounds from Sugarcane Bagasse Hemicellulosic Hydrolysate Treated with Activated Charcoal Before or After Vacuum Evaporation. Brazilian Journal of Chemical Engineering, 2001,18, 299-311.

Rodrigues R.C.L.B., Felipe, M.G.A., Almeida e Silva, J.B., Vitolo, M. Response Surface Methodology for Xylitol Production from Sugarcane Bagasse Hemicellulosic Hydrolysate Using Controlled Vacuum Evaporation Process Variables. Process Biochemistry, 2003.

Sene, L., Vitolo, V., Felipe, M.G.A., Silva, S.S. Effect of Environmental Conditions on Xylose Reductase and Xylitol Dehydriogenase Production in Candida guilliermondii. Applied Biochemistry and Biotechnology, 2000, 84-86, 371-380.

Uhari, M., Tapiainen, T., Kontiokari, T. Xylitol in Preventing Acute Otitis Media. Vaccine, 2001, 19, S144-S147.

Ylikahri, R. Metabolic and Nutritional Aspects of Xylitol. Advances in Food Research, 1979, 25, 159-180. 